\title{
On the influence of the coronal hole latitude and polarity on the geomagnetic activity and cosmic ray variations
}

\author{
Abunina Maria \\ Pushkov Institute of Terrestrial Magnetism, Ionosphere and Radio wave propagation (IZMIRAN) \\ Kaluzhskoe Hwy 4, Troitsk, Moscow, 142190, Russia \\ E-mail: abuninaeizmiran.ru

\section{Abunin Artem, Belov Anatoly, Eroshenko Evgeniya, Gaidash Sergey, Oleneva Victoria, Yanke Victor} \\ Pushkov Institute of Terrestrial Magnetism, Ionosphere and Radio wave propagation (IZMIRAN) \\ Kaluzhskoe Hwy 4, Troitsk, Moscow, 142190, Russia \\ E-mails: abunineizmiran.ru, abeloveizmiran.ru, erosh@izmiran.ru, \\ gaidasheizmiran.ru, oleneeizmiran.ru, yanke@izmiran.ru
}

\section{Kryakunova Olga ${ }^{1}$}

Institute of Ionosphere

Kamenskoe Plato, Almaty, Kazakhstan

E-mail: krolganikeyandex.kz

\begin{abstract}
Changes of geomagnetic activity indices and cosmic ray density and anisotropy variations in high-speed streams of a solar wind from various solar coronal holes are studied. More than 300 coronal holes observed in 2002-2013 were divided into groups taking into account their polarity and heliolatitude. It is shown that transequatorial holes, northern holes with negative polarity and the southern holes with positive polarity are more effective than other holes in creation of geomagnetic activity. The behavior of vector anisotropy of the cosmic rays (especially its North -South component) depends substantially on polarity of a magnetic field in a high-speed streams from the coronal holes. The obtained results have to be useful to forecasting of geomagnetic activity and diagnostics of large-scale interplanetary disturbances.
\end{abstract}

The 34th International Cosmic Ray Conference

30 July- 6 August, 2015

The Hague, The Netherlands

${ }^{1}$ Speaker 


\section{Introduction}

Coronal holes (CHs) - is one of two (along with CME) main solar sources of geomagnetic disturbances $[1,2]$ and transient disturbances of cosmic rays (Forbush decreases). To predict the effects of coronal holes is easier than the effects of CME, as coronal holes live long and change slow. Therefore, exposure time of high-speed solar wind stream on the Earth from a certain coronal hole can be predicted in advance with high accuracy (sometimes even a month in advance). It is more difficult to forecast the geoefficiency of a coronal hole. To predict the expected impact of the high-speed stream on geomagnetic activity is necessary to know its speed and Bz-component of the interplanetary magnetic field. For successful forecasting calculations of the magnetic field on the surface of the solar wind source (eg., [3]) and the area of the $\mathrm{CH}$ (eg., [4]) are used. It is natural to expect that the Bz-component, and therefore the geomagnetic activity, is associated with heliolatitudes and polarity of coronal holes. This connection was examined, for example, in [5], where in addition to the coronal hole heliolatitude the Earth's latitudional position are taken into account.

In this paper we continue to study the effect of coronal holes polarity and heliolatitude on the geomagnetic activity and the CR modulation started in the work [6], significantly expanding the number of events. In addition, in this work we analyze influence of high-speed streams from coronal holes on CR anisotropy.

\section{Data and Methods}

We used the opportunity of the database on the Forbush effects created in IZMIRAN, which contains information on the various parameters of the solar wind, cosmic rays and geomagnetic activity for more than 6700 Forbush decreases (eg. [7]).

For our research we have selected the Forbush decreases, with the sources of high-speed solar wind streams from coronal holes occurred from October 2002 to December 2013, under the following conditions:

- onset of the next event was at least 36 hours from the onset of the analyzed FE;

- onset of the previous events have been at least 60 hours from the onset of the analyzed FE or the magnitude of the previous FE was less than 1.4\%;

- coronal hole polarity was known (negative or positive).

By this way 309 events were selected: 138 holes with positive polarity and 171 with negative. Apparently, we can significantly expand the statistics based on the recently appeared catalog by Tlatov et al. [8].

In our sample there are very large high-speed streams of 2003 with a speed up to $870 \mathrm{~km} / \mathrm{s}$ that Earth passed 6-7 days, and the small one-day streams with a speed below $400 \mathrm{~km} / \mathrm{s}$, which number was a lot in the 2007-2010 solar activity minimum.

The polarity of the coronal hole we determined independently, using the magnetic cards (Wilcox - http://wso.stanford.edu/) and measurements of the solar wind (OMNI http://omniweb.gsfc.nasa.gov). Almost in each case, the polarity coincided with the presented online in http://www.solen.info/solar/.

Figure 1 shows an example of a high-speed stream of positive polarity (By component of the $\mathrm{IMF}>0$ and $\mathrm{Bx}<0$ ). Accordingly, the $\mathrm{CH}$ with a negative polarity had $\mathrm{By}<0$ and $\mathrm{Bx}>0$. 




Figure 1. An example of the solar wind data of a high-speed stream in February 2011. Red line the value of the IMF, green and blue triangles - Bx and By components of the IMF, pink dots Bz component of the IMF, orange dots - SW speed, the vertical bar denotes SSC.

\section{Results and Discussion}

To investigate the effect of high-speed streams from coronal holes on the geomagnetic activity and cosmic rays, taking into account the polarity and location of the holes on the solar disk, we divided the selected coronal holes into 4 groups: 1) coronal holes with negative polarity in the northern hemisphere of the Sun (conventionally denoted «N-»), we had $72 \mathrm{~N}$ - holes; 2) coronal holes with a positive polarity in the southern part of the solar disk ( «S+», 62 holes); 3) transequatorial coronal holes irrespective of the polarity ( «C», 155 holes); 4) those holes that are not in any of the above groups («other», 25 coronal holes). The fourth group got the "wrong" coronal holes, i.e. coronal holes with negative polarity in the southern part of the solar disk, or with a positive polarity in the northern hemisphere. Such holes were a few, as one polarity of the general magnetic field of the Sun prevailed in the studied period.

Figure 2 shows geoeffectivity (maximal $\mathrm{Kp}$ index of geomagnetic activity for all events) of investigated coronal holes and the average $\mathrm{Kp}$-indices for different groups.

We see in Fig. 2 that the most geoeffective group is the transequatorial coronal holes (average $\mathrm{Kp}=4.24 \pm 0.09)$, coronal holes of the first $(\mathrm{N}-)$ and second $(\mathrm{S}+)$ groups $(\mathrm{Kp}=3.92 \pm$ 0.14 , and $\mathrm{Kp}=3.97 \pm 0.12$, respectively) are slightly less geoeffective, and the weakest ones were "untypical" holes $(\mathrm{Kp}=3.16 \pm 0.22)$. This confirms the results of the [6].

These results tell on the impact of the position and polarity of coronal holes on their geoefficiency. But the question arises: whether these groups differ only by latitude and polarity, or they are different also in power? To check how coronal holes geoefficiency depends on the interplanetary characteristics, we used parameter $\mathrm{VmBm}$, which is calculated as follows:

$V m B m=\frac{V_{\max }}{V_{0}} \cdot \frac{B_{\max }}{B_{0}}$, where $V_{0}$ and $B_{0}-$ parameters of undisturbed interplanetary medium (typically used $V_{0}=400 \mathrm{~km} / \mathrm{s}, B_{0}=5 \mathrm{nT}, B_{\max }(\mathrm{nT})$ and $V_{\max }(\mathrm{km} / \mathrm{s})$ - the maximum IMF intensity and the solar wind velocity in the disturbance).

As 1, 2 and 3 groups (N-, S+ and $\mathrm{C}$ ) appeared similar on the effectiveness and were significantly more effective than group 4 , for the study a dependence of the coronal hole geoefficiency on interplanetary characteristics, the first three groups were combined into one 
group NSC, which we compared with the group 4. The results of this comparison are shown in Figure 3.

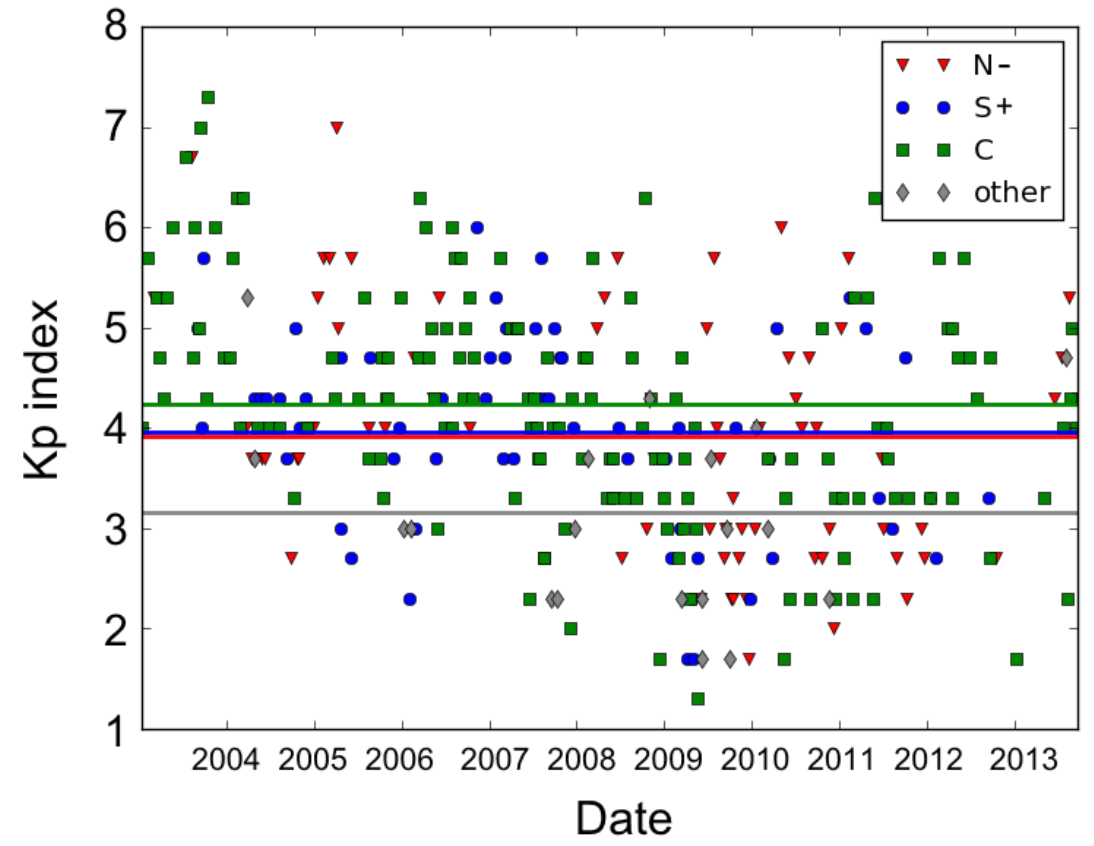

Figure 2. Geoefficiency of coronal holes. Red triangles $(« \mathrm{~N}-»)$ - a group of northern coronal holes with negative polarity, blue circles $(« S+»)$ - Southern positive coronal holes, green squares $(« \mathrm{C} »)$ - transequatorial coronal holes, gray diamonds («other») - "untypical" coronal holes. Green line - average maximum Kp-index transequatorial coronal holes, the blue and red lines are mean values of the maximum Kp-index for the southern positive and northern negative coronal holes, respectively, and gray straight is averaged for the "untypical" coronal holes.

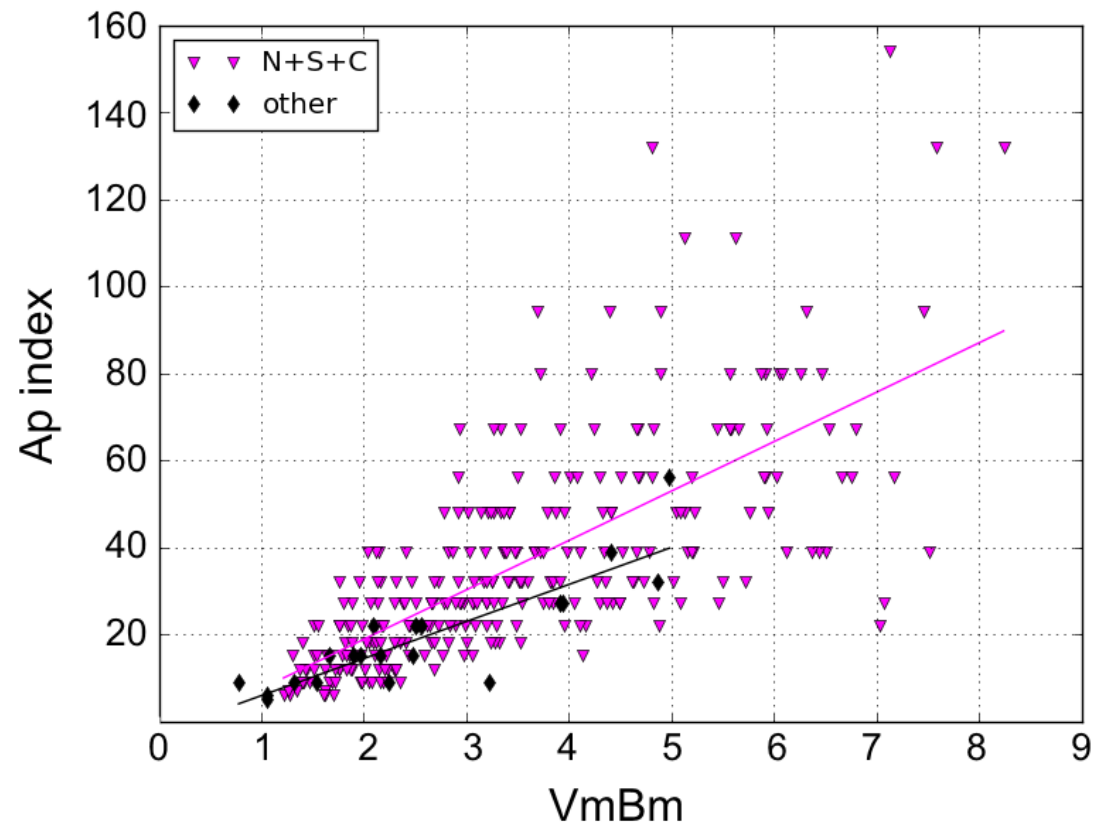

Figure 3. Index of geomagnetic activity Ap depending on the interplanetary characteristics of high-speed streams. Linear regression provides the correlation coefficient $\mathrm{cc}_{\mathrm{NSC}}=0.71$ for more geoeffective holes (pink line), for «untypical» holes $\mathrm{cc}_{\mathrm{other}}=0.86$. 
It can be seen that the holes of low power $(\mathrm{VmBm}<2)$ are similar by efficiency for all groups, but with an increase of power the differences between groups are becoming more visible. For $\mathrm{VmBm}=4$, the difference in the expected Ap-index $\approx 10(2 \mathrm{nT})$. Powerful highspeed streams were absent in "untypical" group. In part this may be due to the limited statistics (in this group only 25 events, significantly less than in the effective united group). Another explanation might be those that untypical holes are associated with the polar hole of the same polarity significantly rarer (which may be a consequence of the fact that in our work mainly a period of one polarity of the total magnetic field of the Sun was considered).

High speed streams affecting the geomagnetic activity, at the same time create an additional modulation of galactic cosmic ray (eg., [9]). Averaged impact of high-speed streams from the holes of different polarity on the behavior of the CR density and anisotropy vector is shown in Figure 4, obtained by the method of superposed epoch.

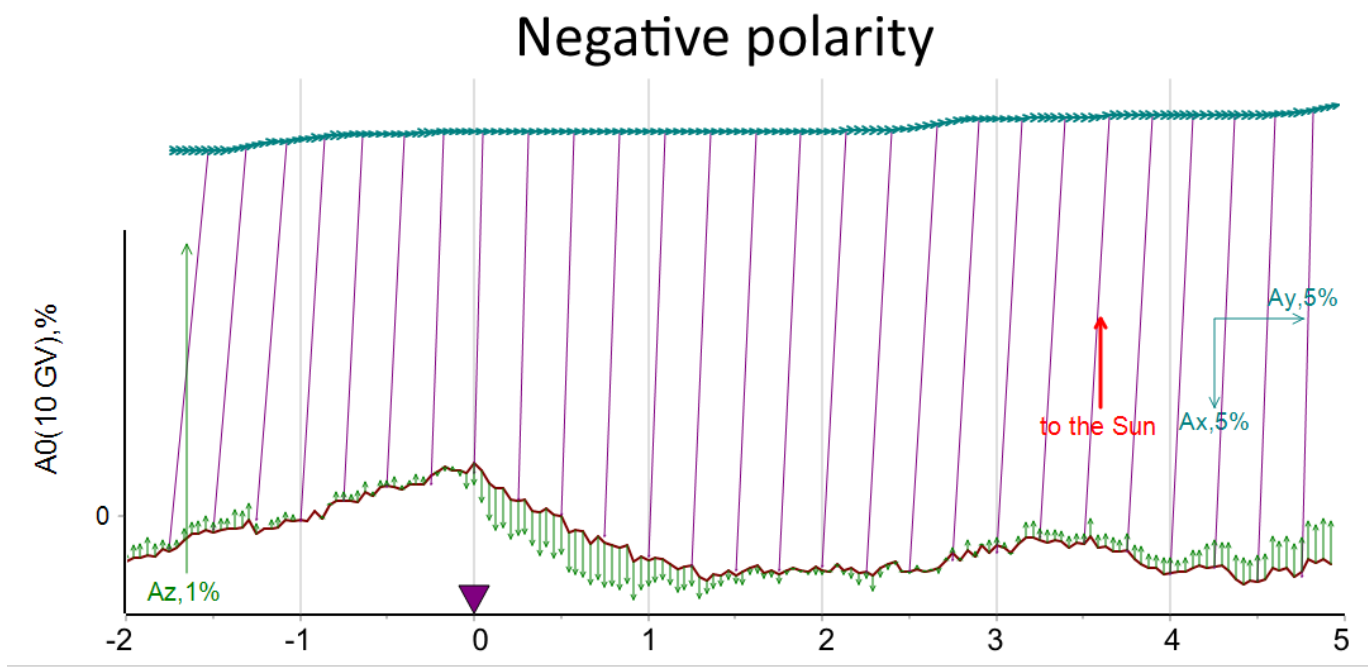

\section{Positive polarity}

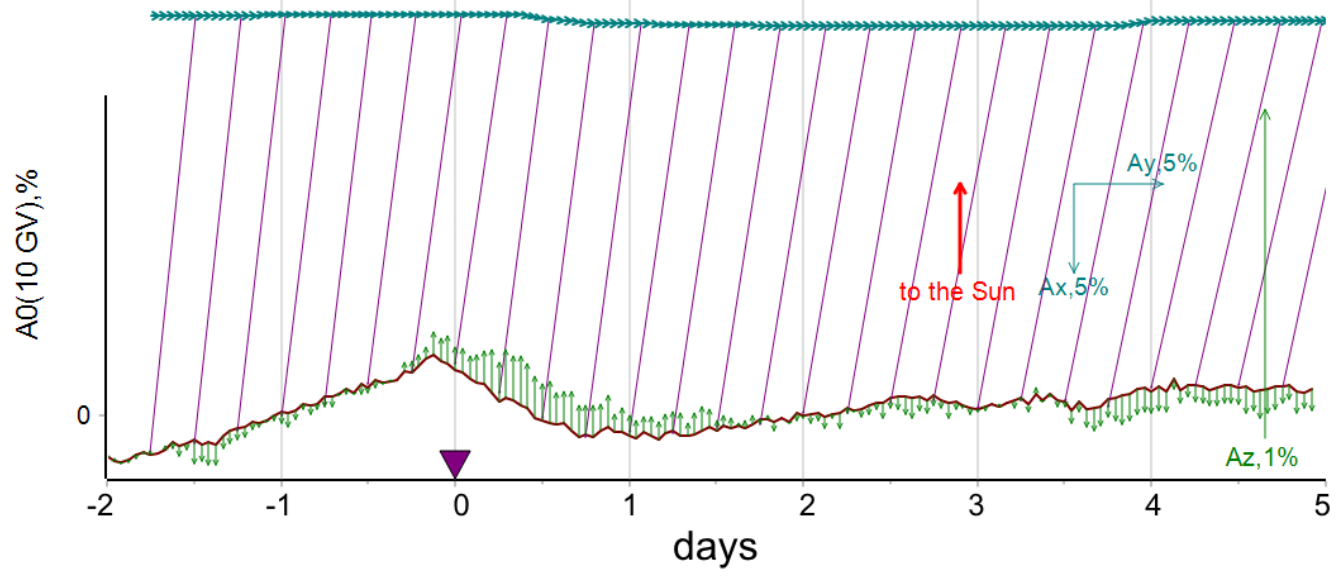

Figure 4. The behavior of the CR density A0 and anisotropy vector in coronal holes with different polarity (superposed epoch). Vertical green arrows correspond to the North-South CR anisotropy. Thin lines connect equal points on time scale on the curves of vector anisotropy and $\mathrm{CR}$ density in each 6 hours. Moment 0 relates to the beginning of the interaction regions of high and low-speed solar wind streams. 
Figure 4 shows that the behavior of the CR density for holes of different polarity is similar and averaged variation of $\mathrm{A} 0$ is low in both cases. Behavior of CR anisotropy is more interesting. In the top panel of Fig. 4 the linked vector diagram shows the behavior of the projection $\overrightarrow{A x y}$ of anisotropy vector on to the plane of Earth's equator. It can be seen that the vector $\overrightarrow{A x y}$ changes quite a little in magnitude and direction during Forbush decreases caused by coronal holes, and this is a significant contrast to its behavior during large FDs caused by $\mathrm{CME}$ (eg. $[10,11])$.

The north-south component $\overrightarrow{A z}$ of the anisotropy directed along the Earth's axis changes much more noticeable, than $\overrightarrow{A x y}$. This component is shown in Fig. 4 as green arrows on the curves of CR density variations. When the Earth comes under the influence of coronal holes, an additional Az-component appears, it directed to the south for holes with a negative polarity and to the north - at the positive polarity.

\section{Main Conclusions}

Coronal holes of the northern hemisphere with a positive polarity and coronal holes of the southern hemisphere with negative polarity are less geoeffective than the holes in the same hemisphere, but of opposite polarity or transequatorial holes. That is expected for excess radial expansion of high-speed streams in which the field lines are deflected to the equator, and the Bz-component of the IMF of different signs is created depending on the position and polarity of the parent hole.

Differences in position and polarity of coronal holes have a little effect on the galactic cosmic ray density variations and equatorial component of anisotropy vector. But the polarity of the holes affects significantly the North-South component of anisotropy - negative polarity reduces it and the positive - increases.

\section{References}

[1] I. G. Richardson, D. F. Webb, J. Zhang, D. B. Berdichevsky, D. A. Biesecker, J. C. Kasper, R. Kataoka, J. T. Steinberg, B. J. Thompson, C.-C. Wu, A. N. Zhukov, Major geomagnetic storms (Dst $\leq-100 \mathrm{nT}$ ) generated by corotating interaction regions. J. Geophys. Res. 111 (2006) A07S09

[2] M. V. Alves, E. Echer, W. D. Gonzalez, Geoeffectiveness of corotating interaction regions as measured by Dst index. J. Geophys. Res. 111 (2006) A07S10

[3] A. Belov, V. Obridko, B. Shelting, Correlation between the near-earth solar wind parameters and the source surface magnetic field. Geomagnetism and Aeronomy 46 (2006) 430-437

[4] Yu. Shugai, I. Veselovsky, L. Trichtchenko, Studying correlations between the coronal hole area, solar wind velocity, and local magnetic indices in the Canadian region during the decline phase of cycle 23. Geomagnetism and Aeronomy 49 (2009) 415-424

[5] Y. Choi, Y.-J. Moon, S. Choi, J.-H. Baek, S. Kim, K.-S. Cho, G. S. Choe, Statistical Analysis of the Relationships among Coronal Holes, Corotating Interaction Regions, and Geomagnetic Storms. Solar Physics 254 (2009) 311-323

[6] M. Abunina, A. Abunin, A. Belov, S. Gaidash, Y. Tassev, P.I.Y. Velinov, L. Mateev, P. Tonev, Geoeffectivity of Solar Coronal Holes with Different Magnetic Field Polarity. Aerospace Res. Bulg. 25 (2013) 70-77 
[7] M. Abunina, A. Abunin, A. Belov, E. Eroshenko, V. Oleneva, V. Yanke. Phase distribution of the first harmonic of the cosmic ray anisotropy during the initial phase of Forbush effects. Proc. 24th ECRS (2015)

[8] A. Tlatov, K. Tavastsherna, V. Vasil'eva, Coronal Holes in Solar Cycles 21 to 23. Solar Physics 289 (2014) 1349-1358

[9] O. Kryakunova, I. Tsepakina, N. Nikolayevskiy, A. Malimbayev, A. Belov, A. Abunin, M. Abunina, E. Eroshenko, V. Oleneva, V. Yanke, Influence of high-speed streams from coronal holes on cosmic ray intensity in 2007. Journal of Physics: Conference Series 409 (2013).

[10] A.V. Belov, Forbush effects and their connection with solar, interplanetary and geomagnetic phenomena, Universal Heliophysical Processes, Proceedings of the International Astronomical Union, IAU Symposium 257 (2008) 439-450

[11] A. Abunin, M. Abunina, A. Belov, E. Eroshenko, V. Oleneva, V. Yanke. Forbush effects with a sudden and gradual onset. Geomagnetism and Aeronomy 53 (2012) 292-299 